\section{NANTES: \\ REGENERACIÓN DEL \\ TERRITORIO INDUSTRIAL \\ Y PLANIFICACIÓN FLEXIBLE}

\section{NANTES: \\ REGENERATING THE \\ INDUSTRIAL TERRITORY \\ AND FLEXIBLE PLANNING}

\author{
Beatriz Fernández \\ Arquitecta. \\ Profesora Asociada de la Escuela Técnica Superior \\ de Arquitectura de Madrid. \\ Universidad Politécnica de Madrid. \\ b.fagueda@upm.es
}

\section{Abstract}

"The present is connected to the future by the possibilities of imagining alternatives; the present is connected to the past because of the necessity of imagining the future as a reelaboration, however fanciful, of what has gone before"

(Sabel et al, 1997)

The changes in the production system and in the organization and division of labor since the second half of the twentieth century have had major impacts on the structure of industrial cities, their social and economic spaces and the lives of workers and people. After an early economic stagnation, most of these regions began to undergo major social and urban declines linked to the loss of their productive base. Since then, there have been many attempts to stop the fall of industrial cities. This article discusses how this deindustrialization process has been dealt with in the region of Nantes, specifically highlighting the links between a new territorial scheme and the rehabilitation of one of the most run down industrial areas in the city: île de Nantes.

\section{Resumen}

"The present is connected to the future by the possibilities of imagining alternatives; the present is connected to the past because of the necessity of imagining the future as a reelaboration, however fanciful, of what has gone before" (Sabel et al, 1997)

Las transformaciones del sistema productivo y los cambios en la organización y división del trabajo ocurridos desde la segunda mitad del siglo XX han tenido repercusiones fundamentales en la estructura de las ciudades asentadas sobre la producción industrial, en su espacio social y económico, así como en las vidas de sus habitantes y trabajadores. Gran parte de estas regiones, tras un inicial estancamiento económico, comenzaron a sufrir importantes procesos de deterioro urbano y social vinculados a la desaparición de su base productiva. Muchos han sido, desde entonces, los intentos de detener el declive de la ciudad industrial. Estudiaremos, en las páginas que siguen, la forma en que la región de Nantes ha afrontado el proceso de desindustrialización, destacando especialmente los vínculos entre la configuración de un nuevo modelo territorial y la rehabilitación de uno de las áreas industriales más deteriorados de la ciudad: île de Nantes.

Palabras clave: urbanismo, territorio, ciudad industrial, planificación, regeneración.

Key words: urbanism, territory, industrial city, planning, regeneration.

Recepción: $\quad 30$ Septiembre de 2011.

Aceptación: 15 Enero de 2012.

\section{Nantes: evolución de la ciudad y declive del modelo industrial}

La evolución de Nantes está marcada por la creación de un espacio económico en torno a la desembocadura del río Loira. Situada en una encrucijada, entre la vía fluvial que organiza el eje este - oeste y la estructura de caminos en sentido norte - sur, Nantes pronto se configuró como ciudad portuaria y espacio de intercambio.

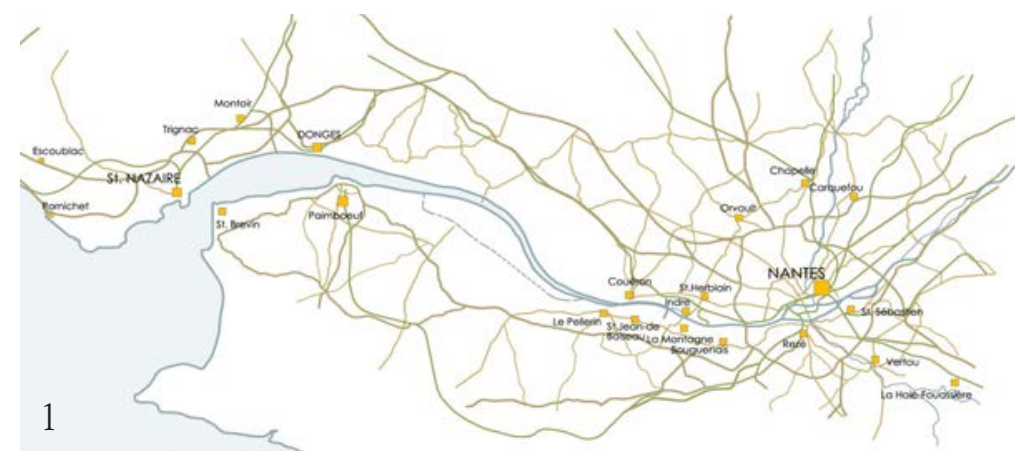

Imagen 1_Nantes en el estuario del Loira (fuente: elaboración propia)

El incremento del transporte marítimo contribuyó al desarrollo del tejido productivo y durante el siglo XIX Nantes se consolidó como una ciudad de carácter industrial destacada en el sector agroalimentario y naviero. El crecimiento de éste último impulsó el desarrollo de la industria química y metalúrgica, que ocuparon las islas del Loira y, de un modo diseminado, su orilla norte desde Nantes hasta su desembocadura en Saint Nazaire. Así se construyó todo un espacio económico lineal a lo largo del Loira que asociaba los dos polos de mayor actividad.

En 1900, más de 30.000 obreros trabajaban en la región, la mayor parte de ellos empleados en la metalurgia y la construcción naval; este crecimiento alcanzó su auge tras la Segunda Guerra Mundial. Sin embargo, el incremento de la competencia, las dificultades de acceso al puerto de Nantes, la crisis económica de los años setenta y el traslado progresivo de las actividades portuarias a Saint Nazaire, provocaron en Nantes unas iniciales dificultades económicas que progresivamente se transformaron en un ciclo caracterizado por el descenso del número de trabajadores en la construcción naval, las fusiones entre empresas y finalmente, su cierre productivo.

Los habitantes y trabajadores comenzaron a sufrir las consecuencias tanto en la reducción del empleo como en el deterioro progresivo de sus barrios. Si bien aparentemente la ciudad conseguía recuperar su base económica gracias al sector servicios, se produjo un desajuste entre la cualificación de gran parte de la población y los nuevos empleos, provocando procesos de empobrecimiento de la población y deterioro económico y social. Los principales emplazamientos de la industria fueron abandonados y espacios centrales de la ciudad se convirtieron en zonas en baldío. Así como el espacio económico de la industria se había construido en torno al Loira, el río, las áreas abandonadas junto a él por la industria y los barrios obreros situados en sus márgenes comenzaron a simbolizar el paro y la crisis de la región. De un modo singular, los espacios anteriormente ocupados por la industria naviera en Île de Nantes, una isla situada frente al centro urbano, se constituyeron en emblema de una ciudad cuyo futuro había sido puesto en entredicho. 
Así, se apostó por una ciudad de la proximidad y del transporte público, basada tanto en la decisión de buscar un modelo más sostenible y duradero como en la convicción de que un mejor acceso a los servicios y espacios de trabajo mejoraría la cohesión social y la calidad de vida.

La articulación entre movilidad y urbanismo y la importancia del transporte público se materializó tanto en un plan a escala regional (Plan de déplacements urbains) como en el sistema de tranvía de Nantes. Los objetivos de esta nueva red iban más allá de la movilidad y convirtieron al tranvía en eje de la transformación urbana, integrando barrios de vivienda social aislados, poniendo en valor espacios públicos en torno a las líneas y conectando con la red de ferrocarril regional.

El plan regional proporcionó las directrices para materializar el modelo territorial, que después se detalló en los planes municipales pero también "se apoya en los proyectos urbanos de regeneración como motor del desarrollo"(ibid). El objetivo era recuperar los espacios centrales de la ciudad industrial como nuevas centralidades a distintas escalas reforzadas por el transporte colectivo. Es decir, frente a las operaciones tradicionales de renovación urbana, la regeneración se abordó desde una perspectiva global que contempló no sólo la preservación del patrimonio industrial como hito o la rehabilitación de los barrios degradados sino la reactivación de los espacios fundamentales de la ciudad industrial en la construcción del futuro de la ciudad.

El polo más importante es Île de Nantes, una isla de 350 ha, que contaba con 13.000 habitantes y 9.000 empleos. Estaba dividida en cuatro grandes zonas: la zona oeste vinculada a las navieras y a la actividad industrial, casi totalmente en baldío, la parte central, formada por barrios obreros en parte deteriorados, la zona este, constituida por un barrio de bloque abierto de la década de 1960 cuya forma de implantación lo aislaba del resto de la trama y el extremo este, una reserva natural.

Si bien durante las dos primeras legislaturas de Jean-Marc Ayrault no se plantearon intervenciones, sí fue un tiempo para establecer prioridades y estrategias, así como los objetivos para el concurso: el tratamiento de la isla como una unidad y el respeto a la memoria del lugar (Masboungi, 2003). Île de Nantes era un territorio complejo de tejidos yuxtapuestos que no parecían tener nada en común. El reto era retomar los vínculos que la decadencia había destruido.

La modalidad de concurso, el marché de définition (Masboungi, 2010), fue muestra del compromiso de la corporación municipal de implicar a toda la sociedad nantesa en el futuro de la isla. Durante un año, los tres equipos invitados definieron el programa de forma progresiva, a través de reuniones con todos los grupos, entendiendo el diálogo como forma de enriquecer el proyecto. El objetivo era que la construcción de la isla fuera un proceso colectivo.

\section{El plan-guide de Île de Nantes: la reactivación de las trazas en la planificación del futuro del territorio}

El proyecto ganador fue, precisamente, el que más puso en valor las trazas del territorio en la planificación del futuro de la isla. Alexandre Chemetoff propuso el plan-guide de île de Nantes, un plan que se aleja por completo de los documentos urbanísticos al uso pues no constituye un proyecto cerrado sino una estrategia de desarrollo territorial en el tiempo. Al tratarse de una actuación a largo plazo, con un horizonte de más de veinte años, el plan-guide se plantea cómo dar coherencia al conjunto sin limitar las posibilidades futuras. Chemetoff lo describe como "un método de trabajo crítico, una planificación relativa, que permita construir la ciudad con todo aquello que la constituye" (Chemetoff, 2010). El plan-guide tomó lo existente como punto de partida, ese état des lieux al que continuamente se refiere Chemetoff, para proponer un itinerario posible de transformación del territorio.

Sin embargo, el "estado del lugar" está en continuamente sometido al cambio y el plan-guide encontró el modo de que el plan evolucionara en función de las transformaciones. El plan-guía fue redibujado trimestralmente durante diez años para incorporar las modificaciones y proponer nuevos escenarios a partir del diálogo con los agentes implicados, adaptándose a las nuevas necesidades y propuestas. Cada revisión era considerada como una hipótesis de desarrollo en un momento determinado. El plan-guide constituye así, un instrumento flexible, capaz de adaptarse a las transformaciones de la ciudad en el tiempo; en otras palabras: un modo de planificar en evolución.

Sin embargo, nada más lejos de su intención que dejar al azar el futuro de la isla. El plan definió la estructura urbana, articulando una red de espacios libres compleja con una estructura parcelaria basada en el tejido existente, recobrando las trazas del pasado de la isla en los nuevos trazados. La trama de espacios públicos es la que permite reconstruir los vínculos que el deterioro destruyó y dar coherencia a los diversos tejidos de Île de Nantes. El espacio público es la estructura que permanece, que organiza lo que existe y lo que puede llegar a ocurrir. A través del tratamiento del espacio público, el plan-guide induce dinámicas de reciclaje, integración y transformación de lo existente; se aleja de la restauración del elemento singular para construir el futuro de la ciudad a partir de aquello que ya está allí, considerando "el estado del lugar como fuente de recursos"(Chemetoff, 2010). El espacio público se ha convertido en Île de Nantes en modelo y referencia de una nueva forma de hacer ciudad, que progresivamente ha sido asumida por los agentes que la construyen. Sobre el espacio parcelado privado el plan-guide no planteó una zonificación al uso sino una normativa flexible, que permitiera dar forma urbana a la diversidad característica de la isla. El plan definía una faisabilité, es decir una de las múltiples posibilidades de hacer, una hipótesis abierta planteada en un momento dado pero cuyo programa podía transformarse en el tiempo, en función de las propuestas y de la propia evolución de la ciudad.

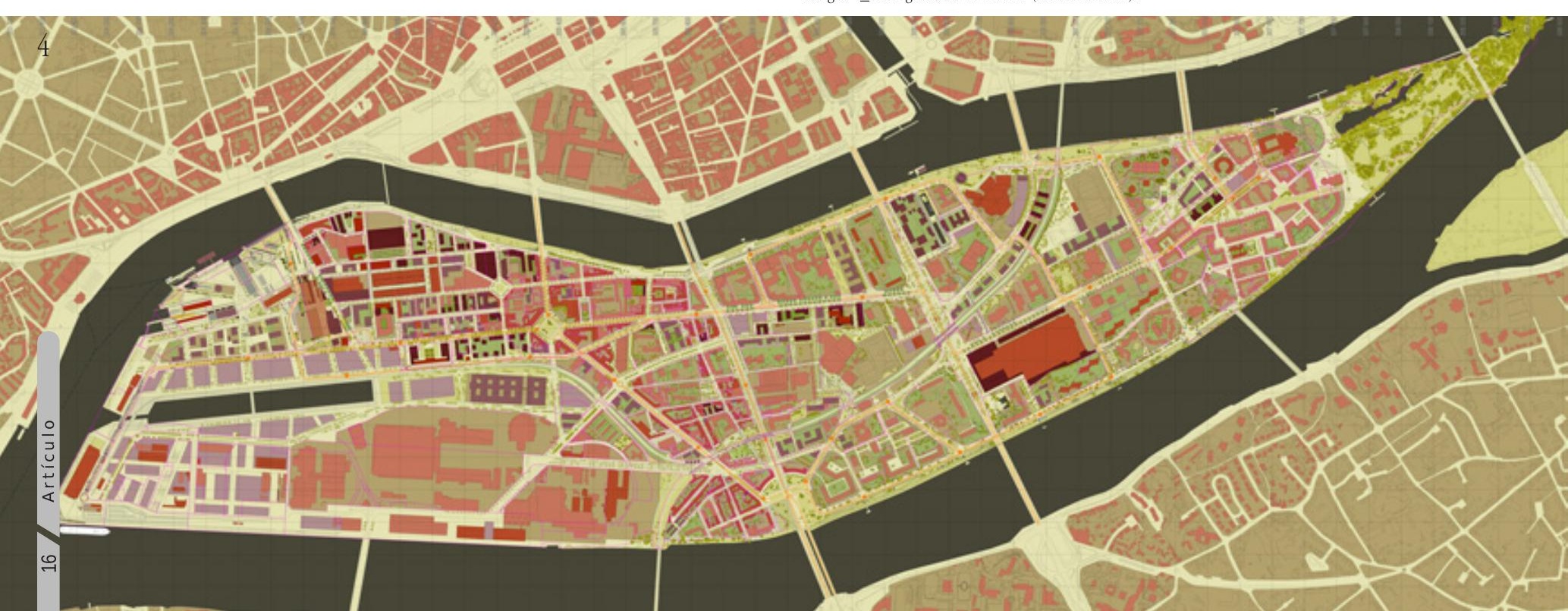


